\title{
Measuring the Deceleration Parameter of the Fission Fragments in a Gaseous Environment for Uranium Coating the Laser-Active Element of the Laser with Nuclear Pumping
}

\section{O. F. Kuharchuk ${ }^{1}$ and L. V. Pityk ${ }^{2}$}

1JSC "Russian Federation State Research Center - Physics and Power Engineering Institute of A.I.Leypunskogo (JSC SSC RF - IPPE), sq. Bondarenko b.1, 249033, c. Obninsk, Kaluga region, Russia

${ }^{2}$ Obninsk Institute for Nuclear Power Engineering of the National Research Nuclear University MEPhl, Studgorodok 1, Obninsk, Kaluga region, 249040, Russia

\section{Abstract}

Corresponding Author:

L. V. Pityk

pityk.leonid@yandex.ru

Received: 23 December 2017

Accepted: 15 January 2018

Published: 21 February 2018

Publishing services provided by Knowledge $\mathrm{E}$

(c) O. F. Kuharchuk and L. V. Pityk. This article is distributed under the terms of the Creative Commons Attribution License, which permits unrestricted use and redistribution provided that the original author and source are credited.

Selection and Peer-review under the responsibility of the AtomFuture Conference Committee.
In this paper the results of the practical definition of the braking parameter of uranium coating fission fragment in the active laser element of nuclear-pumped laser in a gaseous environment are presented. A description of the experimental method for removing the fission spectra of a NPL uranium coating is given. This method is based on measuring the fission fragments energy spectra emitted from the coating to a gas mixture with the help of a semiconductor detector. To calibrate the fission fragment sensors a californium source the fragments of which have a characteristic spectrum is used. The results of the analysis of the sections of the experimental spectra are presented. On the basis of the findings the theoretical model of the fission energy transfer process in a gaseous environment were checked. As the determining parameters in the modeling of this process, the pressure of the gas mixture in the cuvette with the source and the distance from the fission fragment source to the semiconductor sensors are chosen. The functional dependences of the obtained amplitude spectra on these parameters are determined. A mathematical description of the disperse energy fragment process is presented. The basis of this description is a two-parameter dependence.

Keywords: Nuclear-pumped lasers, nuclear-induced plasma, the inhibition of the fission fragments 


\section{INTRODUCTION}

In recent years, much attention has been paid to the development of nuclear-pumped lasers as sources of high-power laser radiation for various practical applications. The operation of nuclear-pumped lasers is based on the processes of ionization and excitation of atoms of a laser active medium by charged products of nuclear reactions, which leads to the formation of a so-called nuclear-excited plasma. One of the most promising methods for obtaining a nuclear-excited plasma is the use of fission products of heavy nuclei. An important characteristic of such processes is the magnitude of energy loss by the fission fragment as it passes by the unit of the path. The study of the phenomenon of deceleration of fission fragments was carried out for many years after the discovery of fission of the uranium nucleus. Despite the fact that a lot of experimental work has been devoted to this issue, unfortunately, there is as yet no complete clarity in this matter. This is due to the fact that the process of deceleration of a fission fragment in a medium is a very complex phenomenon. The object of inhibition itself is not defined in the sense that it is possible to speak of the fission fragment and its characteristics as characteristics averaged, as a rule, over the lung group or in the group of heavy fragments. As the fragment moves through the medium, its parameters change: energy, velocity, ion charge. Since the measurement of these quantities occurs usually at low gas pressures, subsequent extrapolation of the obtained results to higher pressures requires justification, since at the same rates the change in the ion charge of the fission fragment occurs differently in media with different densities.

\section{DESCRIPTION FISSION BRAKING IN THE ENVIRONMENT}

When analyzing the literature data, one can find a number of dependencies describing the process of deceleration of fission fragments in the gas medium. To describe the ionization energy losses by the fission fragment, the Bethe formula is used $[1,2]$ :

$$
-\frac{d E}{d x}=3,072 \cdot 10^{-4} \cdot\left(\frac{Z_{\mathrm{eff}}}{\beta}\right)^{2} \cdot\left(\frac{Z_{m}}{A_{m}}\right) \cdot \ln \left(\frac{2 m_{e} v_{q}^{2}}{I}\right),
$$

where $Z_{\text {eff }}$ is the effective charge of the fission fragment, $\beta=v_{q} / c, v_{q}$ is the fragment velocity, $c$ is the speed of light, I is the average ionization potential of the medium atom with the serial number $Z_{m}$ and the mass number $A, T$ is the mass of the electron.

To clarify the physical meaning of the quantity $d E / d x$, multiply expression (1) by the substance density $\rho\left[\mathrm{mg} / \mathrm{cm}^{3}\right]$, the dimension of the quantity $d E / d x$ in this case will 
be expressed in units of $M e V / c m$. Accordingly, $d E / d x$ is the energy lost by the fission fragment as it passes through a substance of a unit length.

For a more accurate description of $d E / d x$, a formula was used which Grizinski [4] obtained within the framework of the classical description of the process of collision of two particles:

$$
\begin{aligned}
-\frac{d E}{d x}= & N \cdot \sum_{k}\left(\left(\left(\frac{n_{k} \cdot q 0}{U_{k}}\right) \cdot f_{v} \cdot\left(\frac{v_{q}^{2}}{v_{q}^{2}+v_{k}^{2}}\right) \cdot \operatorname{Ln}(\alpha)\right.\right. \\
& \left.\left.+\frac{4}{3} \cdot\left(1-\frac{1}{\alpha}\right) \cdot \operatorname{Ln}\left(e+\frac{v_{q}}{v_{k}}\right)\right) \cdot\left(1-\frac{1}{\alpha}\right)\right),
\end{aligned}
$$

When

$$
\begin{gathered}
f_{v}=\left(\frac{v_{k}}{v_{q}}\right)^{2} *\left(\frac{v_{q}^{2}}{v_{q}^{2}+V_{k}^{2}}\right)^{\frac{3}{2}}, \\
\alpha=4 \cdot\left(\frac{v_{q}}{v_{k}}\right)^{2}\left(1+\frac{v-k}{v_{q}}\right) \\
\sigma_{0}=q^{2} \cdot 6.56 \cdot 10^{14} \mathrm{eV}^{2} \cdot \mathrm{cm}^{2}
\end{gathered}
$$

However, in practice, often used two-parametric dependence [6]:

$$
\frac{E(r)}{E_{0}}=\left(1-\frac{r}{L_{0}}\right)^{\alpha} \text { when } r \leq L_{0},
$$

where the parameter $\alpha=\alpha\left(M_{F F}\right) \approx 1-2[6]$, and the dependence on the parameters of the run fragment in the environment $L_{0}=L_{0}\left(E_{0}, M_{F F}, Z_{F F}\right)$ is determined by the empirical Bohr-Wheeler relation [8]. The energy loss of an individual FF fragment in matter relative to the average kinetic energy as the distance travels is determined by the equation:

$$
\frac{\Delta E(r)}{\bar{E}}=\frac{E_{0}}{\bar{E}}\left[1-\left(1-\frac{r}{L_{F F}}\right)^{\alpha}\right]
$$

It should be noted that the available data on the deceleration parameters of the fragments [7-8] have some scatter. This can, in particular, lead to an error of up to $20-30 \%$ when calculating the transverse profile of the energy input in LAEL. Information on the parameters of inhibition can be obtained by comparing the spectra of fission fragments recorded during braking in a gas medium of different pressures.

\section{DESCRIPTION OF EXPERIMENTAL INSTALLATION AND EXPERIMENTAL METHODOLOGY}

Laser-active element of Stand " $B$ " is a thin-walled steel pipe with an external diameter of $50 \mathrm{~mm}$ and a length of $2500 \mathrm{~mm}$, the inner surface of which is covered with a thin 
layer of metallic uranium-235 with an effective thickness of $\sim 5 \mu \mathrm{m}$. LAEL is sealed from the ends by optical windows and is filled with a laser active medium, which is currently used by He-Ar-Xe (600: 200: 1) mixture with a total pressure of 1 atm.

The operating principle of LAEL is shown in Figure 1:

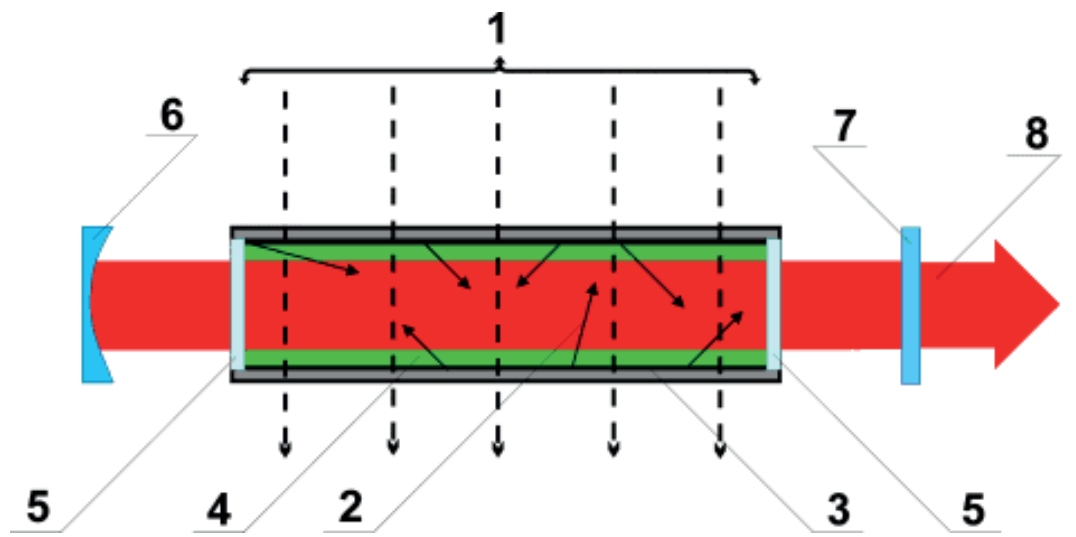

Figure 1: The operating principle of LAEL: 1 - neutron flux; 2 - fragments of divisions; 3 - layer U235; 4 laser active medium; 5 - optical windows; 6 - "deaf" mirror; 7 - output mirror; 8 - laser beam.

Neutrons from the ignition reactor, passing through the laser-active element, cause fission of uranium-235, deposited on the inner surface of the LAEL. Fission fragments, entering the laser-active medium, create a recombination-noequilibrium nuclearexcited plasma and inversion of laser levels. The energy stored in the medium can be extracted from the laser-active element by means of a special optical system consisting of a "dumb" and an output mirror. The laser output characteristics of LAEL with pumping by uranium fission fragments are determined largely by the optical inhomogeneity of the active medium formed during the pumping process. The spatialtemporal inhomogeneity of the optical density of the medium in such a LAEL are mainly due to changes in the gas density due to the nonuniformity along the radius, the angle and the axis of the laser element of the specific energy deposition rate of the fission fragments into the gas, and the presence of heat exchange between the gas and the cuvette wall. These phenomena can lead to the formation of optical in homogeneities such as gas thermolens, an optical wedge and other effects, the magnitude of which, obviously, will depend on the geometry and experimental conditions. Optical inhomogeneity can significantly affect the output power of the laser (the efficiency of the laser optical system) and the divergence of the laser beam. In some cases, the resulting inhomogeneity can lead even to a disruption of laser generation.

The experimental method is based on measuring the energy spectra of fission fragments emitted from the coating to gas (argon), with the help of a semiconductor detector. The fission of uranium is induced by Pu-Be neutrons of a source of intensity 
$8.3 \cdot 10^{6} \mathrm{~s}-1$, slowed down in a polyethylene block. To calibrate the energy scale of the detector, a spectrometrically thin layer of ${ }^{252} \mathrm{Cf}(\sim 50 \mathrm{~s}-1)$ with a diameter of $7 \mathrm{~mm}$ is applied to a substrate of corrosion-resistant steel $0.5 \mathrm{~mm}$ in thickness and $22 \mathrm{~mm}$ in diameter. The number of patches in the coating is determined using a calibrated layer of $\mathrm{U}_{3} \mathrm{O}_{8}$ with a diameter of $30 \mathrm{~mm}$.

The scheme of the experimental setup described above is shown in Fig. 2.

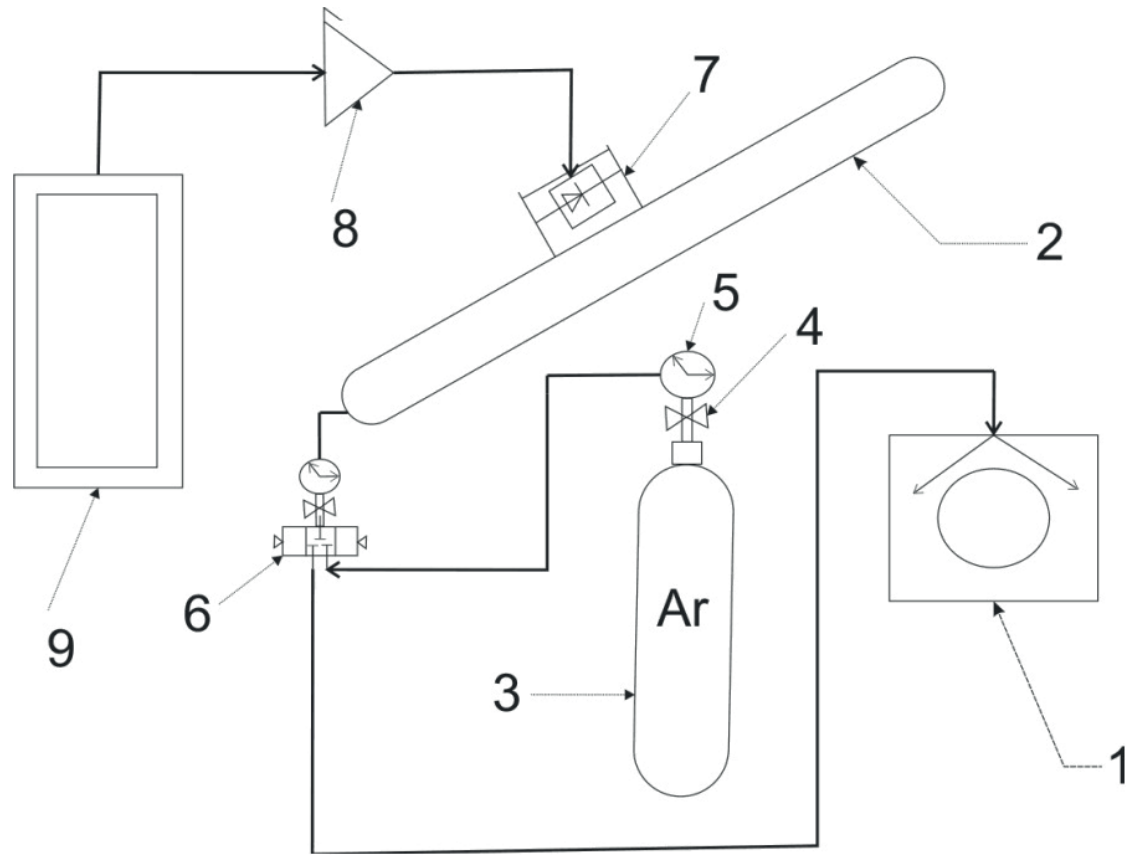

Figure 2: Diagram of the experimental stand: 1 - vacuum pump; 2 - cuvettes with a source; 3 - a balloon with argon; 4 - gas cock; 5 - manometer; 6 - gas distributor; 7 - fission fragment detector; 8 - amplifier; 9 - PC with analog-to-digital converter board.

The main structural element of the installation - a cuvette - a stainless steel pipe with three flanges for installing the detector and applying the test sample. The design of the cuvette had a conclusion for evacuation and gas filling. A fore-vacuum pump $2 \mathrm{HBP}$ carried out the pumping. To measure the vacuum level, a PMT-2 converter and a VIT-2 vacuum gauge were used. Measurement of the gas pressure in the cuvette was carried out using a standard manometer.The branch, located at the center of the main structure, made in the form of a $100 \mathrm{~mm}$ diameter stainless steel pipe, $1 \mathrm{~m}$ long, was installed on the cuvette by flange connection using fluoroplastic gasket. A California source in the form of a "tablet" was placed inside the cuvette to calibrate the unit. As is known, california during the decay gives a characteristic spectrum, which makes it possible to use it for calibration.After calibrating the experimental setup, a uranium layer was placed in the cuvette in the shape and size identical to the California source. Fission fragments are recorded by a silicon surface-barrier detector with a diameter of $25 \mathrm{~mm}$, a thickness of $1.5 \mathrm{~mm}$ with a working surface diameter of $18 \mathrm{~mm}$. 


\section{EXPERIMENTAL RESULTS}

In Fig. 3 shows the amplitude spectra of a uranium-molybdenum coating with a thickness of $10.53 \mathrm{mg} / \mathrm{cm} 2$ at an argon pressure of 0-300 Torr. The spectra contain components corresponding to both fission fragments of uranium (effect) and alpha particles (background).

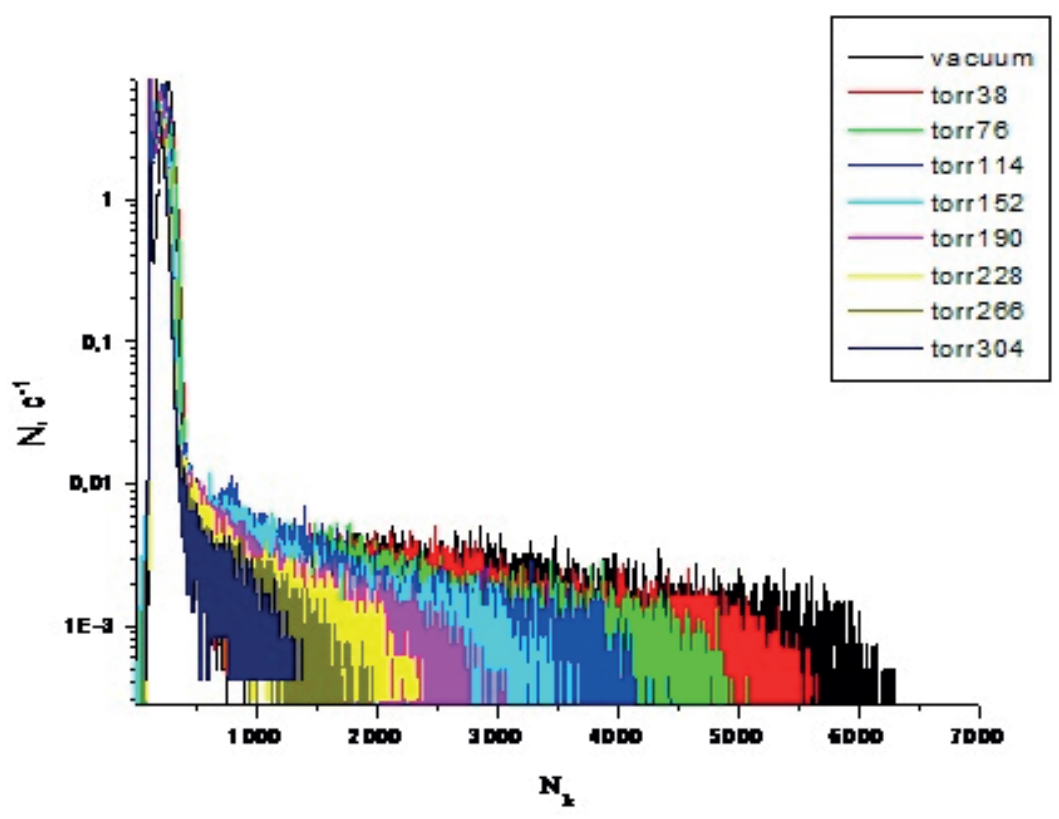

Figure 3: Amplitude spectra of the UM-3 coating recorded at different argon pressures: Nk is the channel number, $\mathrm{N}$ is the logarithmic scale of the number of events per unit time.

To obtain spectra corresponding only to fragments of uranium fission, spectra corresponding to alpha particles (background) were subtracted from spectra containing both components (energy + background).

The calibration scale was also calibrated. The energy calibration of the amplitude scale was carried out in a linear approximation, proceeding from the known values of the most probable energy of the light and heavy fragments of spontaneous fission fragments of ${ }^{252} \mathrm{Cf}$, equal to 106 and $80 \mathrm{MeV}$, respectively [4]. When calibrating, the amplitude-to-digital converter threshold equal to 2.8 channels was also taken into account, and the smallest energy losses of the fission fragments ( $8.2 \mathrm{MeV}$ ) measured in the additional experiment in inert impurities of the californium target used.

Energy spectra of fission fragments were obtained at argon pressure of $0,38,76$, $114,152,190,228,266,304$ torr. As you can see, as the argon pressure increases, the energy spectrum of the particles reaching the detector becomes more and more "shortened". On average, after each change in pressure, the limiting energy of the 


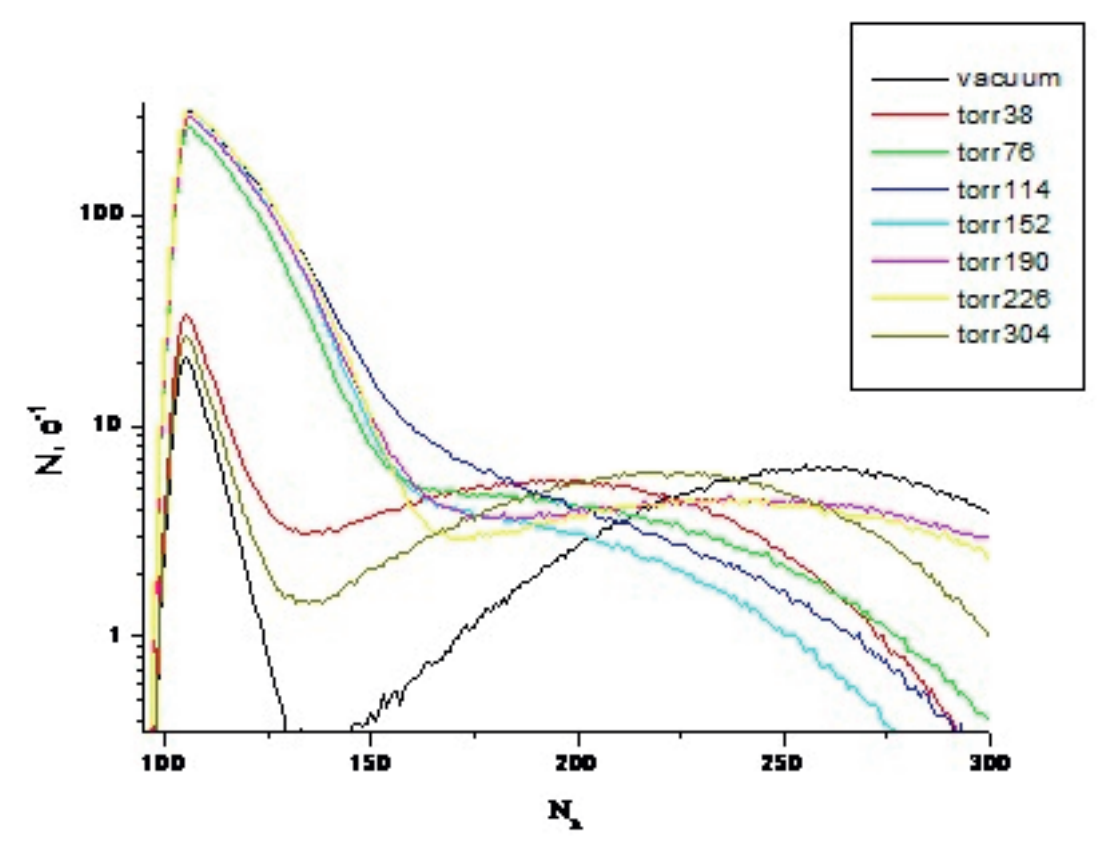

Figure 4: Amplitude spectra of $\alpha$-particles recorded at different argon pressures.

particles decreased by 10-15 Mev. The energy of the $\alpha$ particles was slightly changed, in the course of the whole series of experiments its value was $\sim 7 \mathrm{MeV}$.

\section{EXPERIMENTAL DATA PROCESSING}

The processing of the experimental data consisted of two stages:

1) Analysis of the sections of the experimental spectra and determination of the functional dependences of the obtained data on the initial conditions.

2) Numerical modeling and finding the analytic dependence describing the dissection.

When processing the experimental data, the energy spectra of the fission fragments were used at different pressures.

To obtain the necessary data to determine the experimental dependencies, processing of "raw" data was required. This treatment consisted in determining the limiting value of the energy at which all the experimental data retained the energy integral. The limiting value was taken from the left side of the spectrum.

For a more accurate numerical modeling, two values of the energy integral were chosen: $1.625 \cdot 10^{-2}-1.625 \cdot 10^{-4} \mathrm{MeV} \cdot$ particles. All the investigated intervals of the spectra were at its tail. This is done for greater accuracy and simplification of the 
calculation. In addition, only in the tail of the spectrum, in the region of the fastest particles, all the spectra have approximately the same form. For the processing of "raw" data, a special program has been created that calculates the energy integrals and allows to allocate the necessary energy value. The program is developed using the software package Mathcad 15.

The first stage of data analysis is completed by determining the dependencies between the limiting energy value and argon pressure for each experiment, i.e. for each spectrum. The nature of these dependencies is shown in Fig. 5.

Having a graphical representation of the dependence, it is not difficult to determine the general form of the function describing a given distribution. We repeat the procedure for spectra corresponding to other argon pressures. Numerical modeling is necessary to refine the form of the function and obtain the alpha constant we need. As described above, the particle energy distribution describes a two-parameter exponential function (8):

$$
\frac{E(r)}{E_{0}}=\left(1-\frac{r}{L_{0}}\right)^{\alpha}
$$

For a finite variation, we set $\alpha=1.00$ and build the resulting function in the axes of the experimental data.

We can see that the general form of the function was chosen by us correctly and it is only necessary to change its parameters a little for good agreement with the experimental data.

Thus, the numerical simulation procedure consists in careful selection of the parameter $\alpha$, while the second argument of the function is considered on the entire numerical axis within the boundary conditions until a good agreement with the experiment is obtained. For $\alpha=1.63$, we can see an excellent matching of the values. The variation of $\alpha$ was performed in steps of 0.01 . You can see that the error in numerical simulation is less than $10 \%$.

Because of procedures to refine the coefficient $\alpha$, it was found that $\alpha=1.63$. To obtain the dependencies we need, we change the form of equation (18), multiplying it by $\mathrm{E}_{0}$.

Depending on the distance to the sensor (x), equation (8) will be transformed into a general dependence:

$$
E(x)=E_{0} *\left(1-\frac{x}{L_{0}}\right)^{\alpha}
$$


Depending on the distance to the pressure $(P)$, equation (8) will be transformed into a dependence of the general form

$$
E(p)=E_{0} *\left(1-\frac{p}{p_{0}}\right)^{\alpha},
$$

$\mathrm{P}_{0}$ is the argon pressure at which most of the fragments stop in the near-boundary layer (maximum pressure).

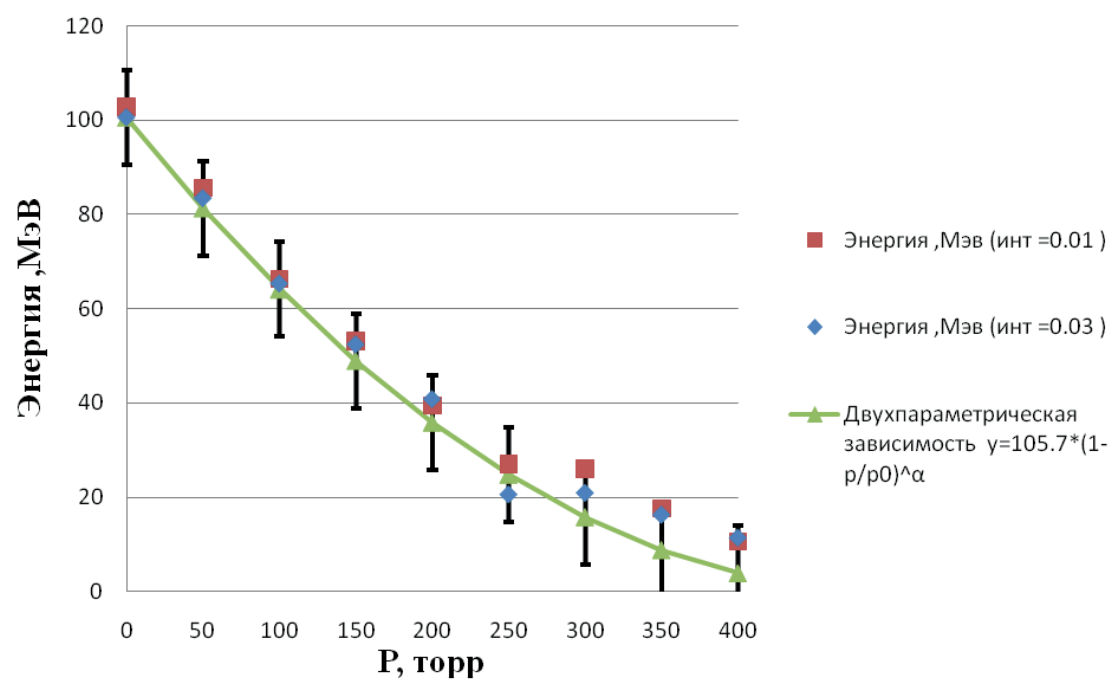

Figure 5: Graph of the dependence of the limiting values of energies on argon pressure.

As can be seen from the graph, the calculated value of the constant $\alpha=1.63$ agrees well with the experimental data

To find $\frac{d E}{d x}$ it is necessary to take the derivative of (8) or (9). Finding the derivative of (9) is not difficult. As a result of the simplest mathematical operations, we obtain the following equations for the energy loss of the fragments:

$$
\frac{d E(x)}{d x}=\frac{-\alpha * E_{0}}{L_{0}} *\left(1-\frac{x}{L_{0}}\right)^{\alpha-1},
$$

\section{CONCLUSION}

Experimental studies of the process of energy loss of fission fragments of the uranium coating of a laser-active element in argon have been carried out. The energy spectra of the fission fragments of uranium-235 at different pressures of the medium are analyzed. The experimental data obtained make it possible to refine the characteristics of the deceleration of fragments of uranium fission in a gaseous medium. The value of the braking parameter $\alpha=1.63 \pm 0.01$ is obtained. The value found does not contradict the available literary data $\alpha=(1-2)$. 


\section{References}

[1] Landau L.D., Lifshitz E.M., Quantum mechanics. M.: Nauka. 1974.2. Rykov V.A. Measurement of non-equilibrium of ionic charge of fission fragments // Preprint FEl-2472. 1995.

[2] Gryzinski M. Classical Theory of Atomic Collisions. I. Theory of Inelastic Collisions //Phys. Rev. 1965. V.138, N 2A. P.336 - 358.

[3] Rvkov V.A., Dyachenko P.P., Mahrov F.V., Socolov Yu.V. Ejected electron energy dependence of ionization cross section of He and $\mathrm{Ar}$ atoms by fission fragments bombardment // Laser and Particle Beams. 1993. V.11,N 3. P.485 - 490.

[4] Physical basics of using the kinetic energy of the fission fragments in radiation chemistry. Ed. Krasin A.K. 1972. Minsk. "Science and Technology".

[5] Reymann K., Schartner K.-H, Sommer B. Scaling relation for total cross section of He in collision with highly charged ions // Phys.Rev. A. 1988. V.5,Vo38. P. 2290-2293.

[6] Betz H.-D., Hortig G., Leischner E., Schmelzer Ch., Staler B., Weihbauch). The average charge of strippe heavy ions // Phys. Lett. 1966.V.5. P.643 - 644.

[7] Grodzins L., Kalish F., Murnick D., Van de Graaff R.J., Chmara F., Rose P.И. Measurements of equilibrium charge states of $\mathrm{Br}, \mathrm{I}$, Ta and $\mathrm{U}$ up to about $200 \mathrm{MeV}$ // Phys. Lett. 1967. V.24B, №6. P.282-285.

[8] Brown M. D., Moak C.D. Stopping powers of some solids for $30-90 \mathrm{MeV}$ U-248 ions // Phys.Rev. 1972. V.6. P.90-94. 